TITLE:

\title{
Two-dimensional space-resolved emission spectroscopy of laser ablation plasma in water
}

\section{$\operatorname{AUTHOR}(S)$ :}

Matsumoto, Ayumu; Tamura, Ayaka; Fukami, Kazuhiro; Ogata, Yukio H.; Sakka, Tetsuo

\section{CITATION:}

Matsumoto, Ayumu ...[et al]. Two-dimensional space-resolved emission spectroscopy of laser ablation plasma in water. Journal of Applied Physics 2013, 113(5): 053302.

\section{ISSUE DATE:}

2013-02-04

URL:

http://hdl.handle.net/2433/187949

\section{RIGHT:}

(C) 2013 American Institute of Physics. This article may be downloaded for personal use only. Any other use requires prior permission of the author and the American Institute of Physics. 


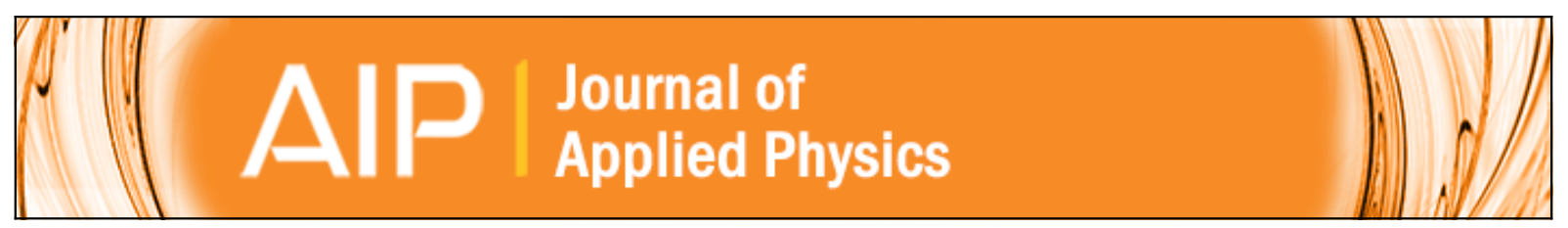

Two-dimensional space-resolved emission spectroscopy of laser ablation plasma in water

Ayumu Matsumoto, Ayaka Tamura, Kazuhiro Fukami, Yukio H. Ogata, and Tetsuo Sakka

Citation: Journal of Applied Physics 113, 053302 (2013); doi: 10.1063/1.4789968

View online: http://dx.doi.org/10.1063/1.4789968

View Table of Contents: http://scitation.aip.org/content/aip/journal/jap/113/5?ver=pdfcov

Published by the AIP Publishing

\section{Articles you may be interested in}

Dynamics of plasma expansion and shockwave formation in femtosecond laser-ablated aluminum plumes in argon gas at atmospheric pressures

Phys. Plasmas 21, 043111 (2014); 10.1063/1.4873701

Emission features and expansion dynamics of nanosecond laser ablation plumes at different ambient pressures J. Appl. Phys. 115, 033107 (2014); 10.1063/1.4862167

Understanding plume splitting of laser ablated plasma: A view from ion distribution dynamics

Phys. Plasmas 20, 113512 (2013); 10.1063/1.4835255

Laser-induced breakdown spectroscopy of tantalum plasma

Phys. Plasmas 20, 073104 (2013); 10.1063/1.4812451

Ultraviolet versus infrared: Effects of ablation laser wavelength on the expansion of laser-induced plasma into one-atmosphere argon gas

J. Appl. Phys. 111, 053301 (2012); 10.1063/1.3689300

\section{AlP Re-register for Table of Content Alerts}




\title{
Two-dimensional space-resolved emission spectroscopy of laser ablation plasma in water
}

\author{
Ayumu Matsumoto, ${ }^{1, a)}$ Ayaka Tamura, ${ }^{1}$ Kazuhiro Fukami, ${ }^{1}$ Yukio H. Ogata, ${ }^{1}$ \\ and Tetsuo Sakka ${ }^{2, \mathrm{~b})}$ \\ ${ }^{1}$ Institute of Advanced Energy, Kyoto University, Uji, Kyoto 611-0011, Japan \\ ${ }^{2}$ Department of Energy and Hydrocarbon Chemistry, Graduate School of Engineering, Kyoto University, \\ Kyoto 615-8510, Japan
}

(Received 26 October 2012; accepted 15 January 2013; published online 4 February 2013)

\begin{abstract}
We developed a method for two-dimensional space-resolved emission spectroscopy of laser-induced plasma in water to investigate the spatial distribution of atomic species involved in the plasma. Using this method, the laser ablation plasma produced on a $\mathrm{Cu}$ target in $5 \mathrm{mM} \mathrm{NaCl}$ aqueous solution was examined. The emission spectrum varied considerably depending on the detecting position. The temperature and the atomic density ratio $N_{\mathrm{Na}} / N_{\mathrm{Cu}}$ at various detecting positions were evaluated by fitting emission spectra to a theoretical model based on the Boltzmann distribution. We are successful in observing even a small difference between the distributions of the plasma parameters along the directions vertical and horizontal to the surface. The present approach gives direct information for sound understanding of the behavior of laser ablation plasma produced on a solid surface in water. (C 2013 American Institute of Physics. [http://dx.doi.org/10.1063/1.4789968]
\end{abstract}

\section{INTRODUCTION}

Laser-induced breakdown spectroscopy (LIBS) is an analytical method based on optical emission spectroscopy of laser-induced plasma. LIBS can be applied for in-situ multielemental analysis of a solid surface in water by measuring an atomic emission spectrum of the laser ablation plasma on a submerged solid target. This technique enables us a realtime composition measurement of the target due to the fast analysis time. Therefore, LIBS has attracted attention as a new technique for resource exploration at sea bottoms. For the application to the seafloor exploration, underwater LIBS analysis of alloys, rocks and sediments has been studied. ${ }^{1-4}$ The measurements under oceanic pressure ${ }^{5-7}$ and downsizing of LIBS instruments ${ }^{8}$ have been reported. A remotesensing of immersed solids in sea has been also performed by mounting the LIBS device on a submarine robot. ${ }^{7}$ This approach considerably reduces the time of exploration, since sample collection from the sea bottom is not necessary.

However, underwater LIBS has a difficulty in shot-toshot reproducibility of the emission spectrum, ${ }^{4,9}$ which has not been improved enough to be applied to quantitative analysis. In order to achieve high reproducibility in the measurement, it is necessary to understand the plasma behavior and to optimize the experimental conditions. The basic researches were performed so far to understand the laser ablation in water ranging from the studies on cavitation bubble, ${ }^{6,10-15}$ irradiation spot, ${ }^{6,11,16}$ particle production in the bubble ${ }^{17}$ to optical emission imaging. ${ }^{18,19}$ It is also important to investigate the spatial distribution of the plasma as well as temporal evolution of the plasma since the plasma distribution is spatially inhomogeneous. However, the spatial distribution of plasma parameters, including the density of the

\footnotetext{
${ }^{a)}$ Electronic mail: matsumto.ayumu.78w@st.kyoto-u.ac.jp.

${ }^{b)}$ Electronic mail: sakka.tetsuo.2a@kyoto-u.ac.jp.
}

atomic species, has not been fully clarified for the laser ablation plasma in water. Emission spectroscopy can be used for the estimation of the plasma parameters, such as temperature, electron density, and atomic density. ${ }^{1,10,20-24}$ Onedimensional distribution of the ablated species from the submerged solid target has been investigated theoretically and also experimentally with the emission spectroscopy. ${ }^{10,25}$ These studies have focused on the distribution in the direction vertical to the target. The plasma emission in water spreads also in the direction horizontal to the target. ${ }^{5,6,8,10,18,19}$ It is absolutely necessary to perform two-dimensional mapping of the plasma parameters for the clarification of the mechanism of the plasma formation. However, the two-dimensional distribution of the atomic species in the laser ablation plasma in water has not been reported, so far. Note that the mapping must be done by a single-shot experiment, since the shot-to-shot variation of the position of the plasma as well as its emission intensity is quite large. Furthermore, the plasma produced on the submerged solid target includes not only the species ejected from the target but also those having been dissolved in surrounding water. ${ }^{1,3}$ The relationship between the ejected species and the dissolved species has not been verified.

In the present study, we developed a method for the two-dimensional space-resolved emission spectroscopy of the laser-induced plasma in water. The plasma in water is confined in a small region and the position of the plasma fluctuates shot-to-shot. This makes the space-resolved emission spectroscopy rather difficult. To perform the spaceresolved emission spectroscopy, we magnified the plasma emission image and detected the light through the optical fibers of which the incident edges were positioned accurately to the image plane. The emission spectra captured by each fiber were measured simultaneously. The image of the plasma was also measured simultaneously to identify the position detected by each fiber. We demonstrate the 


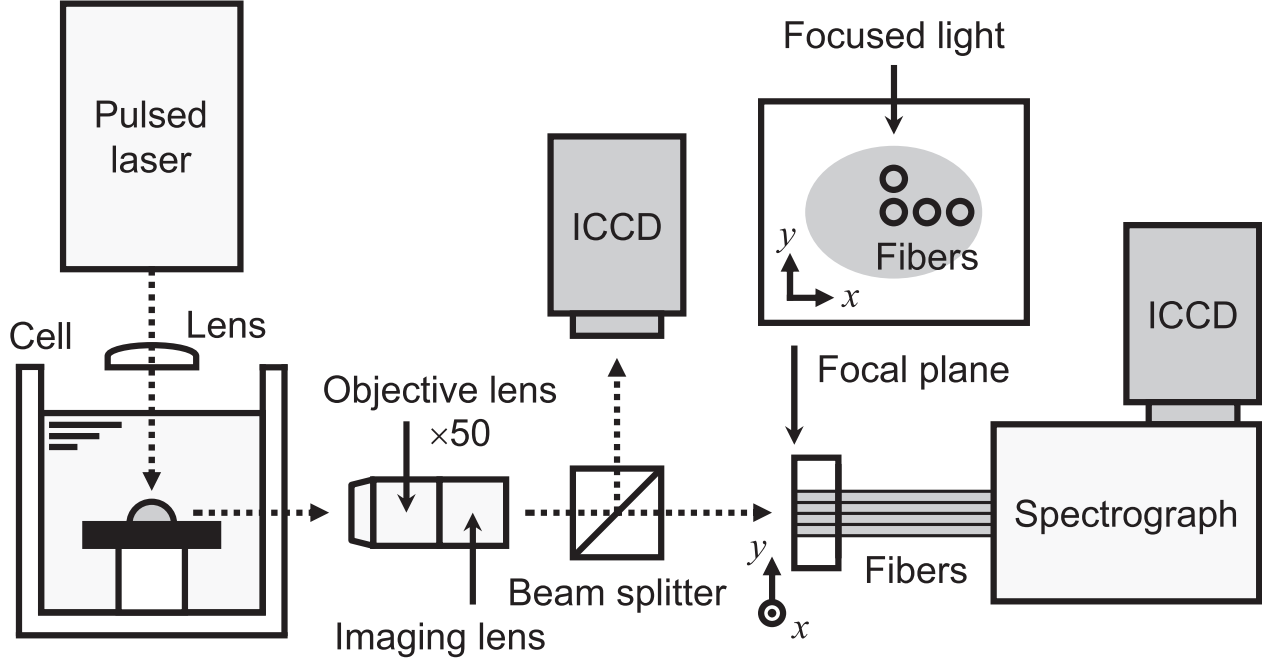

FIG. 1. Experimental setup for twodimensional space-resolved emission spectroscopy of laser-induced plasma in water. The image of the plasma can be measured simultaneously.

Target in solution

space-resolved emission spectra of the laser ablation plasma produced on a $\mathrm{Cu}$ target in $\mathrm{NaCl}$ aqueous solution using the method developed in the present study. We also performed time-resolved emission spectroscopy of the plasma. The temperature and the atomic density ratio $N_{\mathrm{Na}} / N_{\mathrm{Cu}}$ at various detecting positions or various delay times were obtained by fitting the emission spectra to a theoretical model based on the Boltzmann distribution. ${ }^{24}$ To improve the underwater LIBS signal, double-pulse ${ }^{26,27}$ and a single long-pulse $(\sim 100 \mathrm{~ns})^{28}$ irradiation have been proposed. It is important to investigate the plasma produced by the long-pulse laser for the application at the sea bottom, because single pulse ${ }^{7}$ gives clear spectral lines under high pressure environment compared to double pulse. ${ }^{29}$ Therefore, we used the long pulse in the present experiment.

\section{EXPERIMENTAL}

Experimental setup for the space-resolved emission spectroscopy is shown in Fig. 1. A home-built flashlamppumped Q-switched Nd:YAG laser with the fundamental wavelength of $1064 \mathrm{~nm}$, the pulse energy of $6 \mathrm{~mJ}$, the pulse duration of $100 \mathrm{~ns}$ and the repetition rate of $0.3 \mathrm{~Hz}$ was used. The operation procedure to obtain 100-ns pulse duration has been reported. ${ }^{28} \mathrm{~A} \mathrm{Cu}$ plate was placed in a quartz cell filled with $5 \mathrm{mM} \mathrm{NaCl}$ aqueous solution. The distance between the water surface and the target surface was approximately $10 \mathrm{~mm}$. The laser was focused onto the target surface by a 70-mm focal-length plano-convex lens. The plasma emission was 50 times magnified and imaged by using an objective lens together with an imaging lens. After the imaging lens with the focal length of $200 \mathrm{~mm}$, the light was split into two directions by a beam splitter for the measurements of the emission spectrum and the emission image. The entrances of four optical fibers with the core diameter of $910 \mu \mathrm{m}$ (Thorlabs, BFL-22-910) were placed on the focal plane with $1.7 \mathrm{~mm}$ intervals as shown in Fig. 1. The spatial resolution calculated from the size of the fiber entrance is $18.2 \mu \mathrm{m}$. A UV cut filter which blocks the light at the wavelength shorter than $420 \mathrm{~nm}$ was placed in front of the fiber entrance to avoid the detection of the second order diffraction light from UV emission. The light from the exit end of the fiber was introduced to the $100-\mu \mathrm{m}$ slit of a spectrograph (Bunkoukeiki, MK-302) equipped with a 300 grooves/mm diffraction grating. Also, the spectrograph is equipped with an intensified CCD (ICCD) (Princeton, ICCD-1024MTDGE/1) and the emission spectra at each position were simultaneously obtained by using the imaging mode of an ICCD software (Princeton, WinSpec32). An example of the image of the emission spectra is shown in Fig. 2. Clearly separated four bands which line up in the vertical direction are seen in this image. These bands correspond to the emission spectra obtained at each position assigned to each fiber. After data acquisition, binning of 50 pixels in the vertical direction for each band was performed. The sensitivity calibration of the emission spectra at each position was performed with a National Institute of Standards and Technology (NIST)-traceable calibrated quartz tungsten halogen lamp (Oriel, 63355). In order to identify the detecting position, the emission image was obtained simultaneously with the emission spectra by another ICCD (Princeton, PI-MAX: 1K). The emission spectra and the emission images were measured at

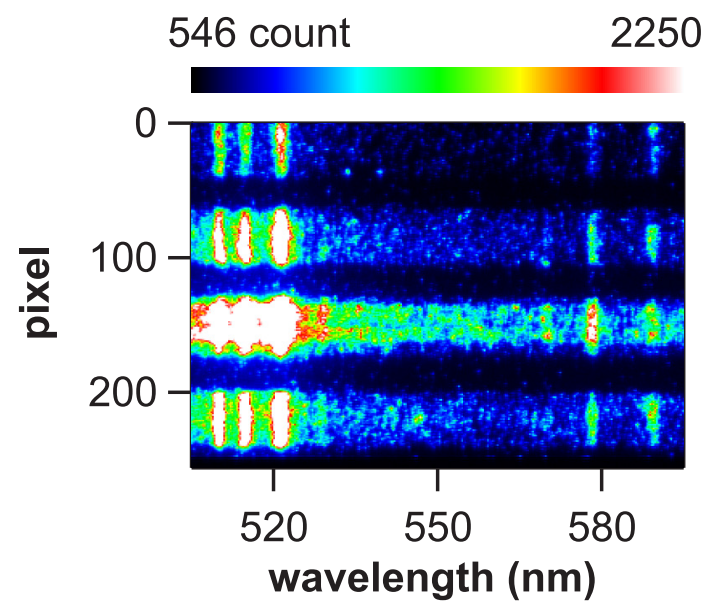

FIG. 2. Example of the spectral image obtained by the space-resolved emission spectroscopy. The image was obtained by the spectrograph, of which four optical fibers are aligned on the entrance slit. Each band of the image corresponds to a single fiber. 
the delay time of $1000 \mathrm{~ns}$ from the laser irradiation to avoid the continuous spectrum of the plasma often observed at the shorter delay times. The gate width of the ICCD was set to 650 ns. A trigger signal was obtained from a PIN photodiode detecting the laser pulse, and the rising edge of the laser pulse was used as the trigger. A shadowgraph image of a cavitation bubble was also measured to investigate the relationship between the plasma emission region and the bubble boundary. The measurement of the shadowgraph image was performed by applying a flashlamp (Morris, Hikarukomachi) as a back illumination light. Except for the back illumination, the setup for the shadowgraph image is the same as that for the emission image shown in Fig. 1. The shadowgraph image was measured at the delay time of $1000 \mathrm{~ns}$ from the laser irradiation. The gate width of the ICCD was set to $10 \mathrm{~ns}$.

In case of the time-resolved emission spectroscopy, the laser with the repetition rate of $1 \mathrm{~Hz}$ was used. The plasma emission was focused onto an optical fiber bundle with the entrance diameter of $1.6 \mathrm{~mm}$ (Oriel, 77532) using two 70-mm focal-length plano-convex lenses. In this case, whole plasma emission imaged by the two lenses enters into the fiber bundle, and hence, the measurement is not spaceresolved. The emission spectrum was obtained by using spectroscopy mode of the ICCD software. The emission spectra were measured at the delay time of 1000, 1250, $1500 \mathrm{~ns}$ from the laser irradiation. The gate width of the ICCD was set to $150 \mathrm{~ns}$. Other experimental conditions were the same as the space-resolved measurement.

\section{DATA ANALYSIS}

In the present study, we assume that local thermodynamic equilibrium (LTE) is satisfied in the laser ablation plasma in water. The Boltzmann plot method has been employed to evaluate the temperature of the plasma in water and giving consistent values. ${ }^{1,2,10}$ The temperature has also been obtained by fitting the continuous spectra to the Plank equation. ${ }^{23}$ Furthermore, rotational and vibrational spectra of $\mathrm{C}_{2}$ molecules obtained by employing a graphite as a target fit well to the theoretical spectra, and the resultant rotational and vibrational temperatures were comparable. ${ }^{20,21} \mathrm{We}$ believe that these studies strongly support the assumption of LTE in the plasma in water.

The emission spectra were fitted to a theoretical model based on the Boltzmann distribution among the levels involved in the related transitions. We neglected selfabsorption effect and assumed that the temperatures of $\mathrm{Cu}$ and $\mathrm{Na}$ atoms are constant. The spectral line profile was approximated by Lorentzian. The formulation is shown below. The profile of emission spectrum $F(\lambda)$ is simply the weighted summation of each normalized form factor

$$
F(\lambda)=\sum_{i} I_{i} f_{i}(\lambda)+C
$$

where $\lambda$ is the wavelength, $I_{i}$ and $f_{i}(\lambda)$ are the intensity factor and the normalized form factor of the spectral line $i$, respec- tively, $C$ is the background correction of the spectrum. ${ }^{24}$ The intensity factor $I_{i}$ is given by

$$
I_{i}=\alpha A_{i} N_{0} \frac{g_{i}}{g_{0}} \exp \left(-\frac{\Delta E_{i}}{k T}\right),
$$

where $\alpha$ is the constant which depends on detection efficiency of measurement system, $A_{i}$ is the Einstein coefficient, $N_{0}$ is the population density of the ground state, $g_{i}$ is the statistical weight of the upper level, $g_{0}$ is the statistical weight of the ground state, $\Delta E_{i}$ is the energy difference between the upper level and the ground state, $k$ is the Boltzmann constant, and $T$ is the temperature. ${ }^{24}$ The form factor $f_{i}(\lambda)$ is assumed to be Lorentzian

$$
f_{i}(\lambda)=\frac{1}{\pi} \frac{\Delta \lambda_{i}}{\left(\lambda-\lambda_{i}\right)^{2}+\Delta \lambda_{i}^{2}}
$$

where $\Delta \lambda_{i}$ and $\lambda_{i}$ are the half width of half maximum and the peak wavelength, respectively. ${ }^{24}$

Followings are the details of the fitting process. The emission spectrum in the spectral range from 500 to $600 \mathrm{~nm}$ was fitted to Eq. (1). We employed $\mathrm{Cu}$ spectral lines at $510.6,515.3,521.8,522.0$ and $578.2 \mathrm{~nm}$, Na spectral lines at 589.0 and $589.6 \mathrm{~nm}$ to be included into Eq. (1). In the fitting process, $\alpha N_{0, \mathrm{Cu}}, T, \Delta \lambda_{i}, N_{0, \mathrm{Na}} / N_{0, \mathrm{Cu}}$, and $C$ were employed as fitting parameters. We also employed $\lambda_{i}$ as a fitting parameter because the precision of the wavelength calibration of the spectrograph is limited, and a small discrepancy of the peak wavelength from the database value may lower the precision of the fitting. The difference in the energies of the upper levels of the $\mathrm{Cu}$ lines allows the estimation of the temperature. Due to the limited resolution of the emission spectrum, the $\mathrm{Cu}$ lines of 521.8 and $522.0 \mathrm{~nm}$ are fitted with a single Lorentzian profile. The $\mathrm{Na}$ lines are also considered to be a single Lorentzian profile. We assumed that the self-absorption effect is negligible, because the $\mathrm{Cu}$ lines are not resonance lines. For the $\mathrm{Na}$ lines, we confirmed the validity of this assumption by measuring the emission spectrum with a higher resolution using a 1200 grooves/mm diffraction grating with keeping the other conditions unchanged. Since the $\mathrm{Na}$ lines (589.0 and $589.6 \mathrm{~nm}$ ) have almost the same value of $A_{i}$ as well as $\Delta E_{i}$, the intensity ratio is determined by the ratio of $g_{i}\left(g_{589.0} / g_{589.6}=2\right)$. If the self-absorption effect was significant, the intensity ratio should have shifted from 2 , because an intense line is more affected by the selfabsorption than less-intense line. However, our experiments show that the intensity ratio of the $\mathrm{Na}$ lines in the present experimental conditions was in good agreement with the ratio of $g_{i}$. Therefore, we assumed that the self-absorption effect of the $\mathrm{Na}$ lines is also negligible although the $\mathrm{Na}$ lines are resonance lines. The parameters other than those employed as the fitting parameters were obtained from the literature. ${ }^{30}$ We obtained the atomic density ratio $N_{\mathrm{Na}} / N_{\mathrm{Cu}}$ from the population density ratio $N_{0, \mathrm{Na}} / N_{0, \mathrm{Cu}}$ using the following relationship:

$$
\frac{N}{N_{0}}=\frac{Z(T)}{g_{0}}=\frac{1}{g_{0}} \sum_{j} g_{j} \exp \left(-\frac{E_{j}}{k T}\right),
$$




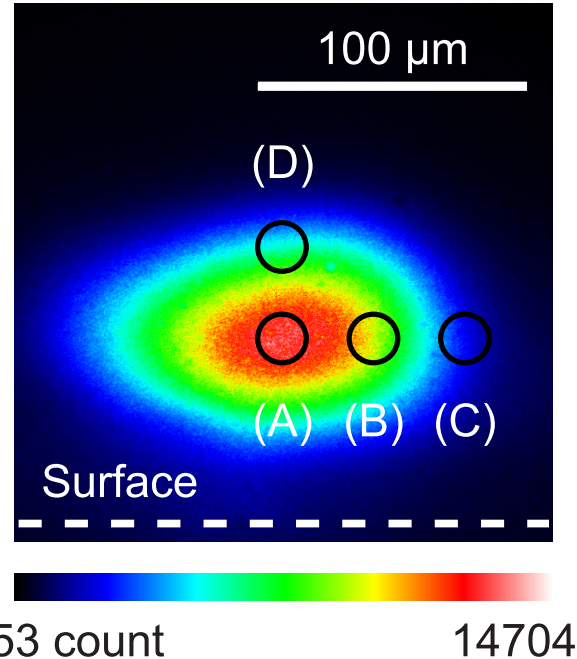

FIG. 3. Emission image of the laser ablation plasma produced on a $\mathrm{Cu}$ plate in $5 \mathrm{mM} \mathrm{NaCl}$ aqueous solution. The pulse width was $100 \mathrm{~ns}$ and the pulse energy was $6 \mathrm{~mJ}$. The emission image was measured at the delay time of $1000 \mathrm{~ns}$ from the laser irradiation. The gate width of the ICCD was set to $650 \mathrm{~ns}$. The circles (A)-(D) show the detecting positions where the emission is detected by the four fibers.

where $Z(T)$ is the partition function, $g_{j}$ and $E_{j}$ are the statistical weight and energy of the level $j$, respectively. $Z(T)$ was obtained from the literature. ${ }^{31}$ Furthermore, we evaluate the relative atomic density of $\mathrm{Cu}$ at each position from $\alpha N_{0, \mathrm{Cu}}$ assuming that $\alpha$ value are the same for all the positions, as long as we are dealing with the spectra obtained by a singleshot irradiation. The relative density of $\mathrm{Na}$ at each position was also obtained.

\section{RESULTS AND DISCUSSION}

The emission image of the plasma and the position where the emission is detected by the fiber are shown in Fig. 3 . The position of the plasma emission fluctuates shot-toshot. Therefore, we selected a position where the emission intensity was highest, and made it the reference point labeled

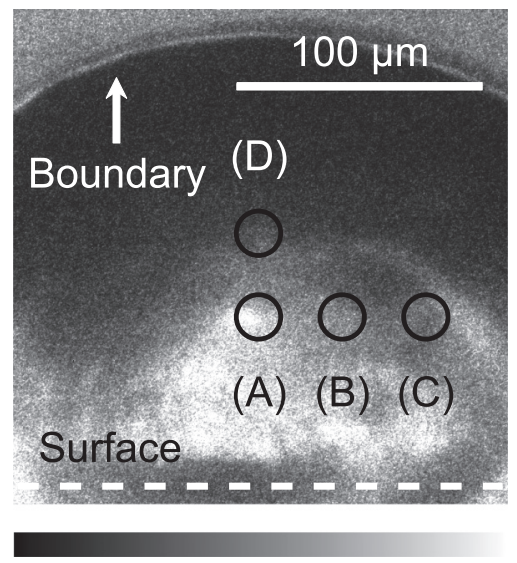

64 count 360

FIG. 4. Typical shadowgraph image of the cavitation bubble produced on a $\mathrm{Cu}$ plate in $5 \mathrm{mM} \mathrm{NaCl}$ aqueous solution. The pulse width was $100 \mathrm{~ns}$ and the pulse energy was $6 \mathrm{~mJ}$. The shadowgraph image was measured at the delay time of $1000 \mathrm{~ns}$ from the laser irradiation. The gate width of the ICCD was set to $10 \mathrm{~ns}$. The spots of detection (A)-(D) are marked. with (A). The other sampling positions are shown in the figure. A typical shadowgraph image in which the spots of the detection are marked is shown in Fig. 4. The plasma emission is not obviously seen in the shadowgraph image since the gate width of the detection is short. By comparing Figs. 3 and 4, it is obviously seen that the plasma emission arises inside the bubble, and the plasma and the bubble boundaries are separated in space. Similar results have been obtained in our previous double-pulse experiment, where the clear spectral line is obtained even without time-gated measurement. ${ }^{8}$ The plasma being well-separated from the bubble boundary at the timing of spectral detection would be one of the reasons why the clear spectral lines can be obtained in the present long-pulse irradiation.

The emission spectra and the best-fit results at each position of detection are shown in Fig. 5. The spectra are wellreproduced by the theoretical spectra described in Sec. III. The spectral lines of $\mathrm{Cu}$ and $\mathrm{Na}$ are simultaneously observed within a single frame of the spectrum. The observation of $\mathrm{Cu}$ and $\mathrm{Na}$ indicates that the plasma includes not only the species ejected from the target but also those dissolved in water, and also that the species originated from the solution are inside the bubble, since the plasma is in the bubble at the timing of the detection. The spectral line of the dissolved species could be used as an internal standard for quantitative analysis of the solid surface in water.

In Fig. 5, we see that the emission intensity of each line varies significantly depending on the position. The intensity ratio of a $\mathrm{Cu}$ line and a $\mathrm{Na}$ line also varies with position. In our previous work, the plasma emission has been introduced into the entrance slit of a spectrograph using a lens system. ${ }^{8,11,16,22,24,28}$ The size of the entrance slit is smaller than the size of the plasma. If the position of the plasma fluctuates shot-to-shot, the position of the image formed at the slit by a lens system would also fluctuate shot-to-shot, and cause a serious intensity fluctuation of the spectra. The experimental setup in the previous works might have some problems in the sampling position. The selection of the detecting position in the plasma is very important for the reproducible measurement.

The temperature and the atomic density ratio $N_{\mathrm{Na}} / N_{\mathrm{Cu}}$ at each detecting position are shown in Fig. 6. The distribution of the temperature is inhomogeneous as shown in Fig. 6(a). The temperature is highest at the center of the plasma and it decreases with distance from the center. Even though the positions (B) and (D) are located at the same distance from the center, the temperature at (B) is higher than that at (D). The present results clearly show that the temperature gradient along the direction parallel to the surface is gentle compared with the direction normal to the surface. Such information cannot be obtained with a combination of vertical and successive horizontal one-dimensional measurements, because the position of the plasma considerably varies shotto-shot, as mentioned above. The result of comparatively gentle temperature gradient along the direction parallel to the surface is related to the oblate plasma as shown in Fig. 3. Such oblateness seems to be due to the finite size of the laser ablation spot of which the diameter is estimated to be from $\sim 50$ to $100 \mu \mathrm{m}$, according to the crater size. The size agrees with the oblateness of the plasma shown in Fig. 3. Also, we 

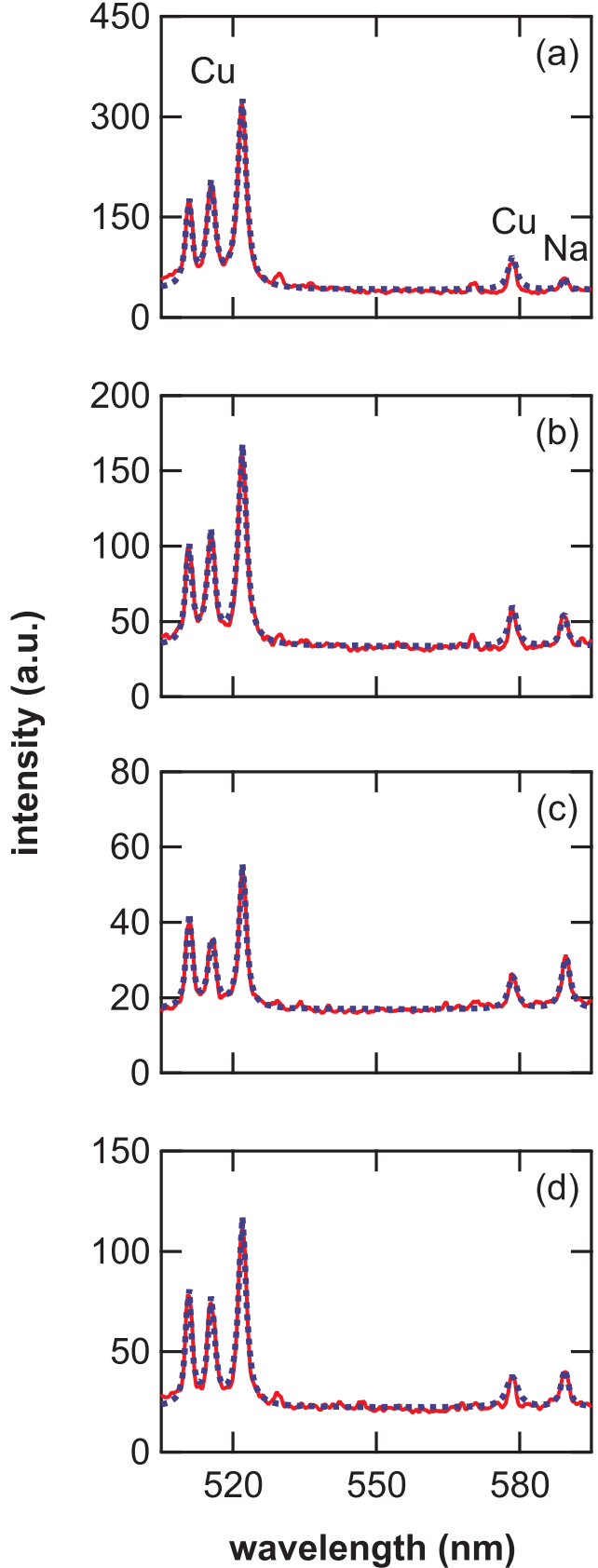

FIG. 5. Emission spectra (solid line) of the laser ablation plasma produced on a $\mathrm{Cu}$ plate in $5 \mathrm{mM} \mathrm{NaCl}$ aqueous solution and the best-fit theoretical spectra (broken line). The results (a)-(d) show the emission spectra obtained at each position (A)-(D), respectively. The pulse width was $100 \mathrm{~ns}$ and the pulse energy was $6 \mathrm{~mJ}$. The emission spectra were measured at the delay time of $1000 \mathrm{~ns}$ from the laser irradiation. The gate width of the ICCD was set to $650 \mathrm{~ns}$.

have observed that the nascent cavitation bubble is not hemispherical but rather flat, ${ }^{32}$ which may help confining the plasma into a flat shape. In Fig. 6(b), we see that the $\mathrm{Cu}$ density is over 1000 times higher than the Na density. Therefore, the distribution of the emission intensity in Fig. 3 is mostly attributed to the ejected species. The plasma emission extends more in the horizontal direction which is parallel to the target surface and the brightest part of the emission is not located immediately on the target surface as shown in Fig. 3. These results are in good agreement with the previous results. ${ }^{18,19}$ The density ratio $N_{\mathrm{Na}} / N_{\mathrm{Cu}}$ is lowest at the center
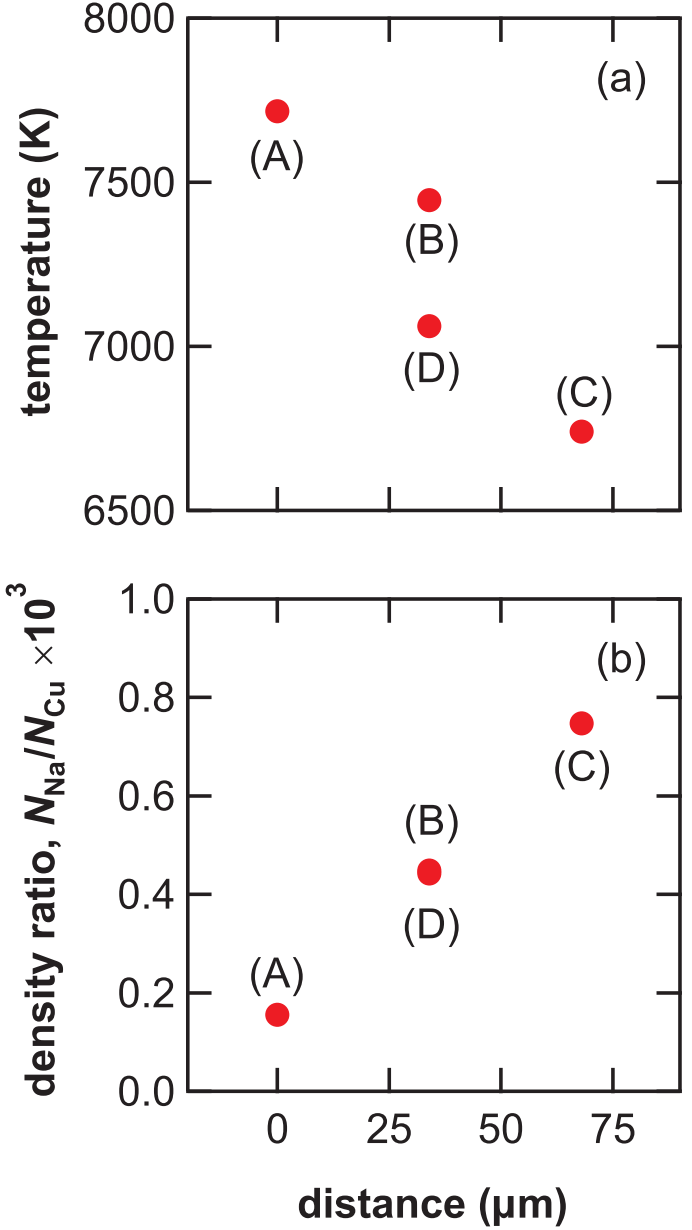

FIG. 6. Temperature (a) and atomic density ratio $N_{\mathrm{Na}} / N_{\mathrm{Cu}}$ (b) obtained as the best-fit parameters at each position (A)-(D). The distance is measured from the center (A).

and it increases with distance from the center as shown in Fig. 6(b). Figure 7 shows the relative density of $\mathrm{Cu}$ and $\mathrm{Na}$ at each position. They are normalized to the value of (A). The $\mathrm{Cu}$ density is highest at the center and it decreases with distance as shown in Fig. 7(a), clearly showing that the distribution of the ejected species is inhomogeneous. The $\mathrm{Cu}$ density at (D) is slightly higher than that at (B). This might be explained by a stronger confinement effect in the direction vertical to the surface than the direction parallel to the surface. On the other hand, Figure 7(b) shows that the Na density at the edge of the plasma is higher than that at the center. The dissolved species seems to be difficult to enter into the high density part of the ejected species compared to the low density edge part. Although we do not have a concrete explanation of such a distribution of the species originated in water phase, we are considering two possibilities to explain the behavior of the dissolved species. First one is that, the dissolved species enter into the plasma immediately after the plasma generation and expand with the plasma evolution. In this case, the plasma expansion makes the species from the solution concentrate in the periphery of the plasma. Second one is that, the dissolved species enter into the plasma through the bubble boundary and increase with the bubble growth. 

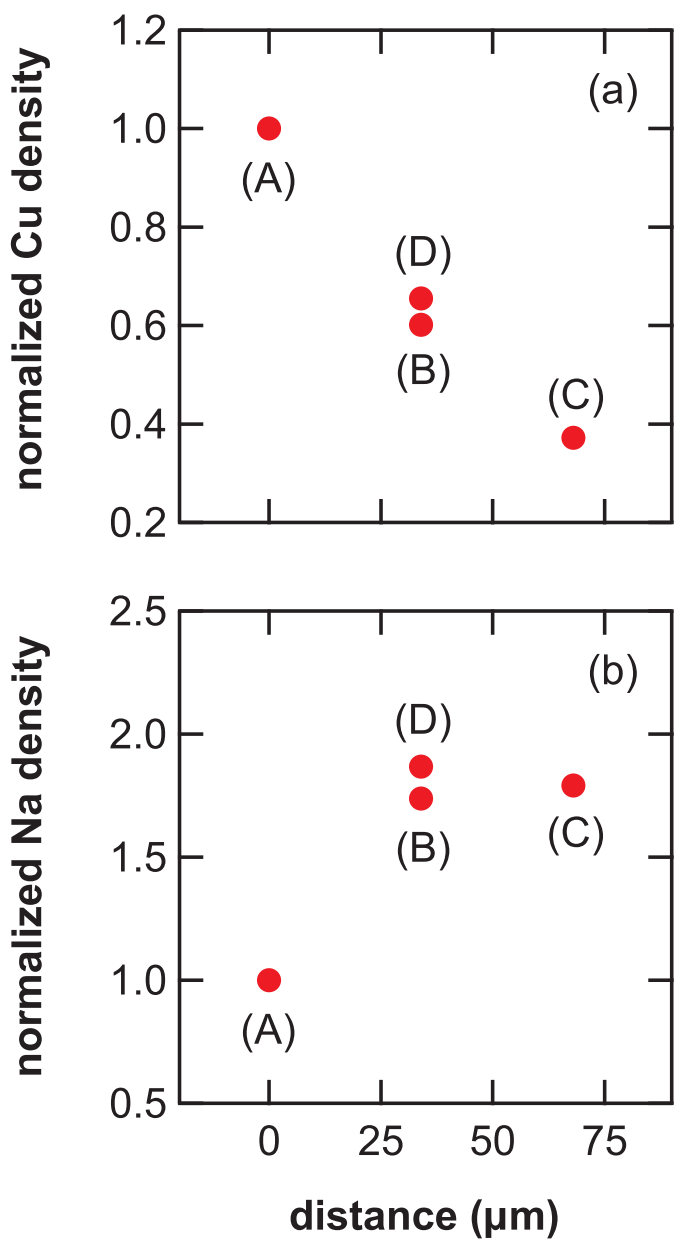

FIG. 7. Relative densities of $\mathrm{Cu}$ (a) and $\mathrm{Na}$ (b) at each position (A)-(D). They are normalized to the value of (A).

In the following time dependence of the emission spectra will be discussed. The two-dimensional space-resolved emission spectra measured at various delay times would be of great value to investigate the detail of the plasma behavior. However, it is difficult to obtain reliable data due to the shot-to-shot fluctuation of the plasma. Therefore, we measured whole plasma without considering the detecting position as described in the experimental section, and 30 spectra were averaged in order to reduce the shot-to-shot fluctuation. The results are shown in Fig. 8. The intensity of each line is strongly dependent on the delay time. The intensity ratio of a $\mathrm{Cu}$ line and a $\mathrm{Na}$ line also varies with time. These results indicate that the delay time should be fixed for the application to the quantitative analysis. Figure 9 shows the temperature and the atomic density ratio $N_{\mathrm{Na}} / N_{\mathrm{Cu}}$ at each delay time. The parameters are the averaged values of the whole plasma emission, and hence, we cannot discuss any spatial distribution of the plasma parameters. The temperature decreases with time as shown in Fig. 9(a). It should be noted that the ratio $N_{\mathrm{Na}} / N_{\mathrm{Cu}}$ obtained by the time-resolved measurement shown in Fig. 9(b) gives higher values compared to that obtained by the space-resolved measurement shown in Fig. 6(b). The detecting area of the time-resolved measurement includes wider area where the ratio $N_{\mathrm{Na}} / N_{\mathrm{Cu}}$ is higher. The ratio $N_{\mathrm{Na}} / N_{\mathrm{Cu}}$ increases with time. The density of each ele-
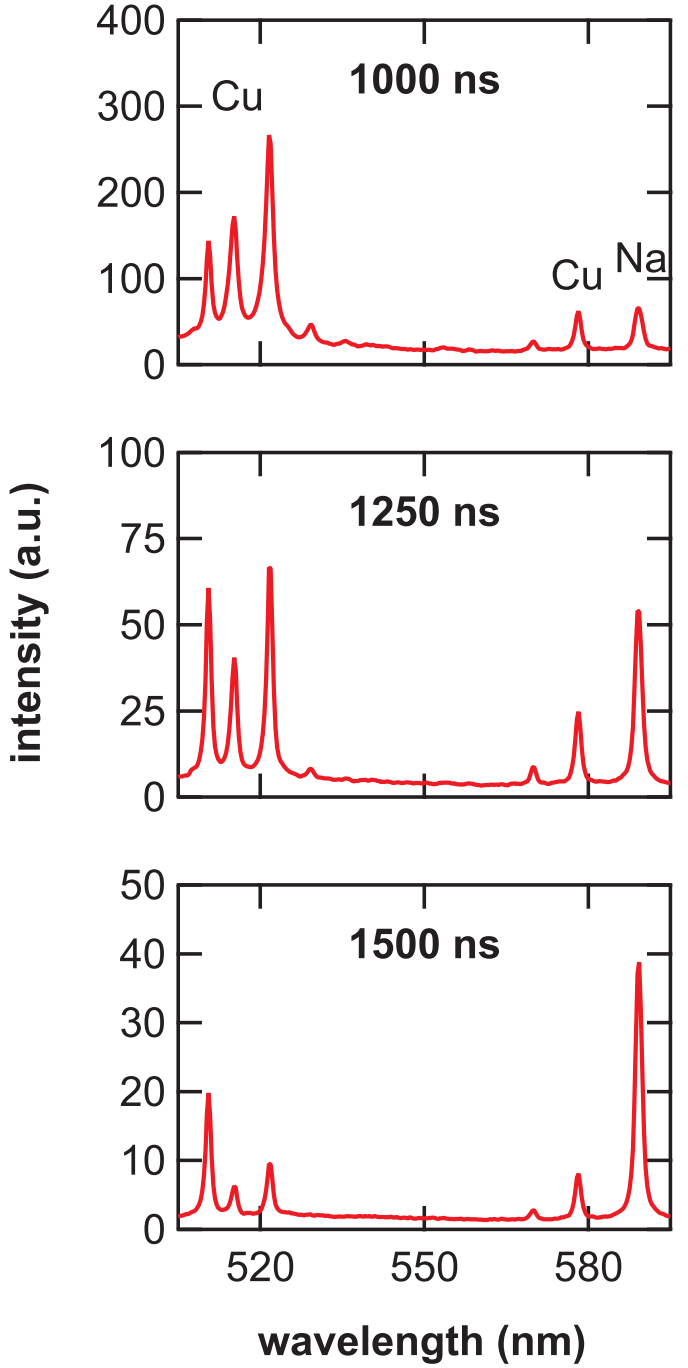

FIG. 8. Emission spectra of the laser ablation plasma produced on a $\mathrm{Cu}$ plate in $5 \mathrm{mM} \mathrm{NaCl}$ aqueous solution at various delay times. The pulse width was $100 \mathrm{~ns}$ and the pulse energy was $6 \mathrm{~mJ}$. The delay times from the laser irradiation were 1000,1250 , and $1500 \mathrm{~ns}$. The gate width of the ICCD was set to $150 \mathrm{~ns}$.

ment relative to a certain delay time cannot be evaluated because we cannot assume that $\alpha$ is the same for all the delay times due to the shot-to-shot fluctuation. However, we are considering two possibilities to explain the result. First one is that, the $\mathrm{Cu}$ atoms are gradually oxidized by the interaction with the surrounding gas in the bubble. ${ }^{10,25}$ Second one is that, the number of $\mathrm{Na}$ atoms increases with the bubble growth, since the dissolved species enter into the plasma through the bubble boundary.

The results obtained in the present work strongly suggest the importance of two-dimensional space-resolved measurement not only for accurate diagnostics of the laser plasma in water but also for the position-selective detection in actual underwater LIBS measurement for spectrochemical analysis. In order to detect the LIBS signal in a positionspecified manner, we believe that the technique based on the present experimental setup is essential, and can be used for the future development of underwater LIBS instrument which measures position-specified spectra. 

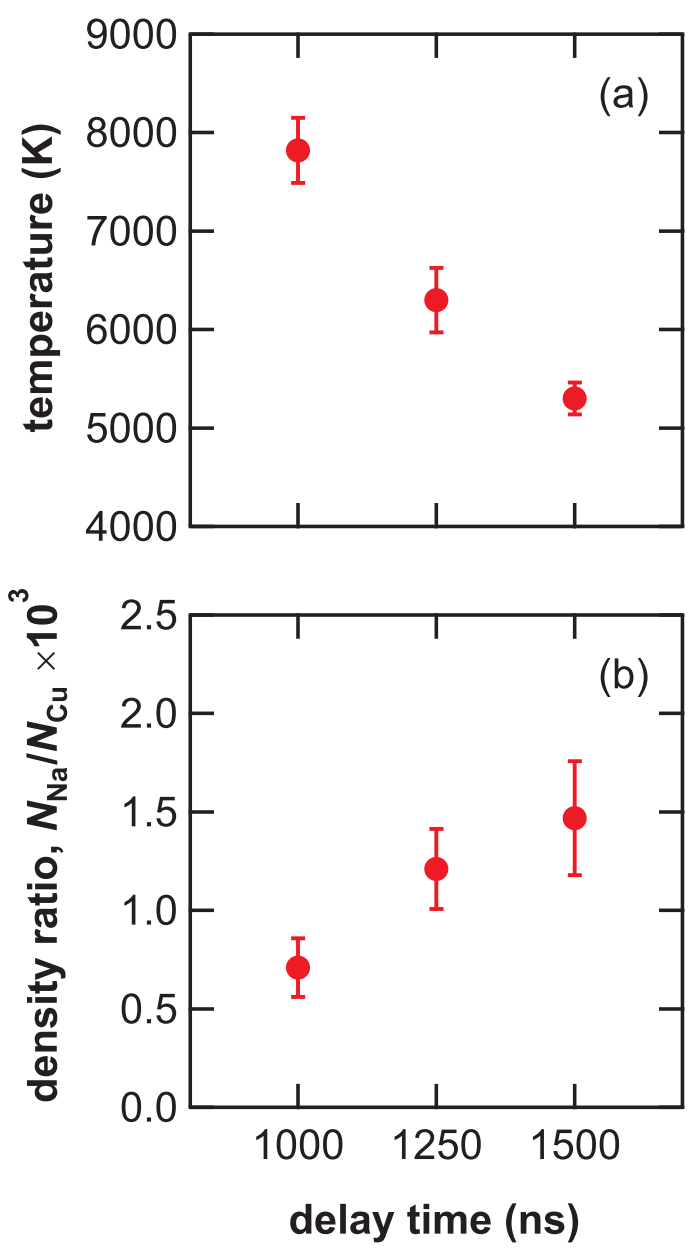

FIG. 9. Temperature (a) and atomic density ratio $N_{\mathrm{Na}} / N_{\mathrm{Cu}}$ (b) obtained as the best-fit parameters. They are plotted as a function of the delay time.

\section{CONCLUSIONS}

In the present study, we developed a new experimental method for two-dimensional space-resolved emission spectroscopy of laser-induced plasma in water. We applied this technique to the space-resolved emission spectroscopy of the laser ablation plasma produced on a $\mathrm{Cu}$ target in $\mathrm{NaCl}$ aqueous solution, and successfully obtained the two-dimensional distributions of temperature and atomic species including not only the species ejected from the target but also those originated from the solution, without being disturbed by shot-toshot fluctuation. The present method of two-dimensional space-resolved emission spectroscopy has a great potential to be used in the detailed investigation of laser ablation plasma in water.

\section{ACKNOWLEDGMENTS}

The present study was financially supported partly by JSPS Grant-in-Aid for Scientific Research (No. 23560023). Also, the present study includes the result of "Development of laser remote analysis for next generation nuclear fuel and applied study by MOX sample" entrusted to Japan Atomic Energy Agency by the Ministry of Education, Culture, Sports, Science and Technology of Japan (MEXT).

${ }^{1}$ A. De Giacomo, M. Dell'Aglio, F. Colao, and R. Fantoni, Spectrochim. Acta, Part B 59, 1431 (2004).

${ }^{2}$ A. De Giacomo, M. Dell'Aglio, F. Colao, R. Fantoni, and V. Lazic, Appl. Surf. Sci. 247, 157 (2005).

${ }^{3}$ V. Lazic, F. Colao, R. Fantoni, and V. Spizzicchino, Spectrochim. Acta, Part B 60, 1014 (2005).

${ }^{4}$ V. Lazic, F. Colao, R. Fantoni, V. Spizzichino, and S. Jovicevic, Spectrochim. Acta, Part B 62, 30 (2007).

${ }^{5}$ B. Thornton and T. Ura, Appl. Phys. Express 4, 022702 (2011).

${ }^{6}$ B. Thornton, T. Masamura, T. Takahashi, T. Ura, T. Sakka, and K. Ohki, Proceedings of IEEE/MTS Oceans'11 Kona, 110416-001 (2011).

${ }^{7}$ B. Thornton, T. Masamura, T. Takahashi, T. Ura, K. Ohki, and T. Sakka, Proceedings of IEEE/MTS Oceans'12, Hampton Roads, VA 120514-002 (2012).

${ }^{8}$ T. Sakka, A. Tamura, T. Nakajima, K. Fukami, and Y. H. Ogata, J. Chem. Phys. 136, 174201 (2012).

${ }^{9}$ V. Lazic, F. Colao, R. Fantoni, and V. Spizzicchino, Spectrochim. Acta, Part B 60, 1002 (2005).

${ }^{10}$ A. Casavola, A. De Giacomo, M. Dell'Aglio, F. Taccogna, G. Colonna, O. De Pascale, and S. Longo, Spectrochim. Acta, Part B 60, 975 (2005).

${ }^{11}$ T. Sakka, S. Masai, K. Fukami, and Y. H. Ogata, Spectrochim. Acta, Part B 64, 981 (2009).

${ }^{12}$ K. Sasaki, T. Nakano, W. Soliman, and N. Takada, Appl. Phys. Express 2, 046501 (2009).

${ }^{13}$ W. Soliman, T. Nakano, N. Takada, and K. Sasaki, Jpn. J. Appl. Phys. 49, 116202 (2010).

${ }^{14}$ G. Cristoforetti, M. Tiberi, A. Simonelli, P. Marsili, and F. Giammanco, Appl. Opt. 51, B30 (2012).

${ }^{15}$ A. Tamura, T. Sakka, K. Fukami, and Y. H. Ogata, Appl. Phys. A "Dynamics of cavitation bubbles generated by multi-pulse laser irradiation of a solid target in water," (to be published).

${ }^{16}$ T. Sakka, H. Oguchi, S. Masai, and Y. H. Ogata, Chem. Lett. 36, 508 (2007).

${ }^{17}$ W. Soliman, N. Takada, and K. Sasaki, Appl. Phys. Express 3, 035201 (2010).

${ }^{18}$ K. Saito, K. Takatani, T. Sakka, and Y. H. Ogata, Appl. Surf. Sci. 197198, 56 (2002).

${ }^{19}$ H. Oguchi, T. Sakka, and Y. H. Ogata, J. Appl. Phys. 102, 023306 (2007).

${ }^{20}$ T. Sakka, K. Saito, and Y. H. Ogata, Appl. Surf. Sci. 197-198, 246 (2002).

${ }^{21}$ K. Saito, T. Sakka, and Y. H. Ogata, J. Appl. Phys. 94, 5530 (2003).

${ }^{22}$ T. Sakka, K. Hirata, S. Masai, and Y. H. Ogata, Proc. SPIE 5713, 276 (2005).

${ }^{23}$ H. Ushida, N. Takada, and K. Sasaki, J. Phys.: Conf. Ser. 59, 563 (2007).

${ }^{24}$ T. Sakka, H. Yamagata, H. Oguchi, K. Fukami, and Y. H. Ogata, Appl. Surf. Sci. 255, 9576 (2009).

${ }^{25}$ A. De Giacomo, M. Dell'Aglio, A. Casavola, G. Colonna, O. De Pascale, and M. Capitelli, Anal. Bioanal. Chem. 385, 303 (2006).

${ }^{26}$ R. Nyga and W. Neu, Opt. Lett. 18, 747 (1993).

${ }^{27}$ A. E. Pichahchy, D. A. Cremers, and M. J. Ferris, Spectrochim. Acta, Part B 52, 25 (1997).

${ }^{28}$ T. Sakka, H. Oguchi, S. Masai, K. Hirata, Y. H. Ogata, M. Saeki, and H. Ohba, Appl. Phys. Lett. 88, 061120 (2006).

${ }^{29}$ A. De Giacomo, A. De Bonis, M. Dell'Aglio, O. De Pascale, R. Gaudiuso, S. Orlando, A. Santagata, G. S. Senesi, F. Taccogna, and R. Teghil, J. Phys. Chem. C 115, 5123 (2011).

${ }^{30}$ P. L. Smith, C. Heise, J. R. Esmond, and R. L. Kurucz, "Atomic spectral line database," in Atomic Line Data Kurucz CD-ROM No. 23, Smithsonian Astrophysical Observatory, edited by R. L. Kurucz and B. Bell (Cambridge, MA, 1995).

${ }^{31}$ See http://www.nist.gov/pml/data/asd.cfm for "Partition Function Can be Obtained by NIST Atomic Spectra Database."

${ }^{32} \mathrm{~T}$. Sakka, (unpublished). 